have argued that it could represent a distinct clinical entity. The present study undertook in depth phenotyping along with assessment of cortical function to further explore disease pathophysiology in MND with malignancy (MND-M) patients.

Methods Clinical features along with assessment of peripheral and cortical function was undertaken in 13 MND-M and results were compared to sporadic and familial MND cohorts. Results From a cohort 13 patients (10 males; aged 65.2 \pm 2.0 years), $30.8 \%$ were diagnosed with a haematological malignancy. The lower motor neuron phenotype predominated in the in the MND-M patients $(\chi 2=10.8, P<0.01)$, with the upper motor neuron (UMN) score being significantly reduced in MND-M patients compared to sporadic and familial MND cohorts $(\chi 2=6.84, \mathrm{P}<0.01)$. The neurological deficits did not respond to treatment of the underlying malignancy in the majority of MND-M (92\%) patients, and as such there were no significant differences in survival between the cohorts. Despite a paucity of UMN signs, cortical hyperexcitability was evident in MND-M patients, as indicated by reduction in short interval intracortical inhibition $(\mathrm{P}<0.01)$ and increase in motor evoked potential amplitude $(\mathrm{P}<0.01)$, that were similar to findings in sporadic and familial MND cohorts.

Conclusions The present study suggests that MND-M falls within the spectrum of MND. A co-incidental association between MND and malignancy is underscored by cortical dysfunction and clinical findings which seems within the spectrum of abnormality evident in classical MND phenotypes.

\section{HOW TO DIAGNOSE LEWY BODY DEMENTIA? PREVALENCE AND UNDERLYING RELATIONSHIP BETWEEN CLINICAL AND NEUROPSYCHOLOGICAL FEATURES OF DLB}

Elie Matar*, Kaylena A Ehgoetz Martens, Glenda M Halliday, Simon JG Lewis. Brain and Mind Centre, University of Sydney, Camperdown, NSW, Australia

\subsection{6/jnnp-2019-anzan.81}

Introduction Despite its importance for management, prognostication and selection of patients for clinical trials, the diagnosis of Dementia with Lewy Bodies (DLB) remains challenging. Complicating this is a recent change in the diagnostic criteria which has arguably shifted the expected phenotype of DLB patients. In this study we aimed to characterize and examine the relationship between cognitive and clinical diagnostic variables in DLB patients to uncover latent symptom clusters that may streamline future diagnostic approaches in the clinic.

Methods The clinical and neuropsychological profile of 27 prospectively recruited participants diagnosed with probable DLB and 25 age-matched controls was characterized according to the most recent consensus criteria.Symptoms were scored using a novel combination of established clinical and research instruments.

Results We demonstrate comparable sensitivity of formal neuropsychological testing and bedside screening tools (MOCA/ MMSE) for identifying domain-specific differences between controls and patients $(\mathrm{p}<0.001)$. Optimal sensitivity thresholds for diagnosis of Parkinsonism (88.9\%) were explored yielding a prevalence range of 50\%-90\% within our cohort.Factor analysis using all core and supportive features of the diagnostic criteria identified 6 independent factors accounting for $81 \%$ of the total variance. Unique relationships identified included between hallucinations and fluctuations and excessive daytime somnolence; between REM sleep behavior disorder and orthostatic hypotension; and Parkinsonism and urinary disturbance. 'Prodromal' symptoms including autonomic and early neuropsychiatric features are represented in the remaining factors.

Conclusion Parsimonious delineation of clinical variables using identified symptom clusters can aid DLB diagnosis.Clusters are also used to highlight latent pathological relationships. Appropriate instruments and thresholds for detecting dementia and core and suggestive features are presented.

\section{TOWARDS OBJECTIVE TESTING IN PARKINSON'S DISEASE: A SYSTEMATIC REVIEW OF THE LITERATURE LOOKING AT ASSESSMENT OF POSTURAL SWAY}

${ }^{1}$ Wenbo Ge*, 2,1 Deborah Apthorp, ${ }^{3,4}$ Christian J Lueck, ${ }^{1,5,6,7}$ Hanna Suominen. 'Research School of Computer Science, The Australian National University, Canberra, ACT, Australia; ${ }^{2}$ School of Psychology and Behavioural Sciences, University of New England, Armidale, NSW, Australia; ${ }^{3}$ Department of Neurology, Canberra Hospital, Canberra, ACT, Australia; ${ }^{4}$ Medical School, The Australian National University, Canberra, ACT, Australia; ${ }^{5}$ Machine Learning Research Group, Data61/CSIRO, The Australian National University, Canberra, ACT, Australia; ${ }^{6}$ Faculty of Science and Technology, University of Canberra, Bruce, ACT, Australia; ${ }^{7}$ Department of Future Technologies, University of Turku, Turku, Finland

\subsection{6/jnnp-2019-anzan.82}

Introduction Parkinson's Disease (PD) is associated with increased mortality and reduced quality of life. There is currently no accurate objective measure for use in diagnosis or assessment of severity. Analysis of postural sway may help in this regard. This systematic review aimed to assess the effectiveness of the various features currently used to analyse postural sway.

Methods Five databases were searched for articles that examined postural sway in both PD patients and controls. An effect size (ES) was derived for every feature reported in each article. The most effective features and feature-families were determined, along with the influence on these measures of data sampling rate and experimental condition.

Results 441 papers were initially retrieved, of which 31 met the requirements for analysis. The most commonly-used features were not the most effective (e.g. PathLength had an ES of 0.47 while TotalEnergy had an ES of 1.78). Decreased sampling rate was associated with decreased ES (e.g. ES of PathLength lowered from 1.12 at $100 \mathrm{~Hz}$ to 0.40 at $10 \mathrm{~Hz}$ ). Being off medication was associated with a larger ES (e.g. ES of PathLength was 0.21 on medication and 0.83 off medication).

Conclusions Some measures of postural sway are better able to distinguish PD patients from controls than others. ES is enhanced by using a higher sampling rate and studying patients off medication. These results will inform future studies looking at postural sway in PD and contribute to the aim of finding an objective marker of the disease.

\section{PREDICTING PARKINSON'S AND DEMENTIA WITH LEWY BODIES (PRE-D) RESEARCH STUDY - A SYDNEY-BASED LONGITUDINAL BIOBANKING PROGRAM}

${ }^{1}$ Elie Matar*, ${ }^{1}$ Kaylena A Ehgoetz Martens, ${ }^{2}$ Maria Comas Soberats, ${ }^{1}$ Glenda M Halliday, ${ }^{1}$ Simon JG Lewis. ${ }^{1}$ Brain and Mind Centre, University of Sydney, Camperdown, NSW, Australia; ${ }^{2}$ Woolcock Institute of Medical Research, Sydney, NSW, Australia

\subsection{6/jnnp-2019-anzan.83}

Introduction Idiopathic REM sleep behaviour(iRBD) disorder represents the most specific prodromal marker of an 
impending synucleinopathy with over 90\% developing either Parkinson's disease(PD), Dementia with Lewy Bodies(DLB) or Multiple System Atrophy(MSA) after 15 years. This finding has stimulated efforts to actively register and track progression of such patients. Here we present experience of a biobanking program established with the aim of identifying prodromal synucleinopathies to facilitate recruitment to neuroprotective trials as they become available.

Methods Patients with iRBD were prospectively and sequentially recruited. Cross-sectional comparator groups consisting of healthy controls, idiopathic PD (within 5 years of diagnosis) and DLB were also recruited. Patients underwent a standardized assessment protocol including clinical phenotyping, neuropsychometric testing, multimodal MRI, polysomnography, quantitative electroencephalography, chronobiology (melatonin and clock gene expression profiling) and gait testing. Subjects were invited for annual and biennial review.

Results 102 patients have been recruited into the study since July 2016 including 35 patients with iRBD, 26 DLB, 19 early PD and 16 controls. 15 patients have returned for follow-up with 3 converting to a synucleinopathy(2 DLB, 1 PD). $75 \%$ of participants were able to complete all elements of assessment protocol. Preliminary evaluation of iRBD participants reveals early changes in clock gene expression (BMAL1) and subtle changes in patterns of gait compared to older controls.

Conclusions Our preliminary findings demonstrate utility and feasibility of a prodromal biobanking program within the Australian context aimed at identifying prodromal synucleinopathies. Similar models can be applied to other centers to improve access and create an extended national collaborative network.

\section{ASSESSMENT OF THE EFFICACY OF ERENUMAB DURING THE OPEN-LABEL TREATMENT (13-24 WEEKS) OF SUBJECTS WITH EPISODIC MIGRAINE WHO FAILED 2-4 PRIOR PREVENTIVE TREATMENTS: RESULTS OF THE LIBERTY STUDY}

\begin{abstract}
${ }^{1}$ Lauren Giles*, ${ }^{2}$ Uwe Reuter, ${ }^{3}$ Peter Goadsby, ${ }^{4}$ Michel Lanteri-Minet, ${ }^{5}$ Peggy Hours-Zesiger, ${ }^{5}$ Chrystel Fernandes, ${ }^{6}$ Michel Ferrari, ${ }^{5}$ Jan Klatt. ${ }^{1}$ Austin Health, Melbourne, VIC, Australia; ${ }^{2}$ Department of Neurology, Charité Universitäts Medizin, Berlin, Germany; ${ }^{3}$ University of California, San Francisco, USA; ${ }^{4}$ Pain Department, Université Côte d'Azur, Nice, France; ${ }^{5}$ Novartis Pharma AG, Basel, Switzerland; ' ${ }^{6}$ Leiden University Hospital, Leiden, Netherlands
\end{abstract}

10.1136/jnnp-2019-anzan.84

Introduction To assess efficacy of erenumab in the first three months of the open-label extension phase (OLEP; 13-24 weeks) of the LIBERTY study.

Methods In the double-blind treatment phase (DBTP), 246 patients were randomized to placebo and erenumab $140 \mathrm{mg}$ for 12 weeks, following which, patients completing that phase $(\mathrm{N}=240)$ were enrolled in OLEP, to receive monthly erenumab $140 \mathrm{mg}$. Outcomes measured monthly throughout to week 24 were achievement of at least 50\%/75\%/100\% reduction in monthly migraine days (MMD), change from DBTP baseline in MMD, monthly acute migraine-specific medication days (MSMD), Headache Impact Test (HIT-6TM) total score, everyday activities (EA) and physical impairment (PI) as measured by the Migraine Physical Function Impact Diary (MPFID).

Results Overall, 228/240(95.0\%) patients completed the 24 week visit of the OLEP. In the overall population at Week
$24,39.2 \%, 15.9 \%$ and $7.0 \%$ patients achieved $\geq 50 \% / \geq 75 \% /$ $100 \%$ reduction in MMD. The mean (standard deviation) change from DBTP baseline in MMD was -2.7(4.4) and $-1.4(3.0)$ in MSMD; and -7.6(8.0), -2.5(9.2) and -4.0 (9.0) in HIT-6TM, MPFID-PI and MPFID-EA scores respectively. Patients with continuous use of erenumab showed sustained efficacy in all outcomes assessed. Patients who switched from placebo to erenumab in the OLEP showed improvement from the first measurement at Week 16 on all outcomes assessed.

Conclusions Efficacy of erenumab was sustained throughout 24 weeks in a hard to treat patient population with multiple prior preventive treatment failures. Overall, efficacy data over 24 weeks (assessed over weeks 13-16,17-20 and 21-24) was generally in line with prior erenumab trials.

\section{ASSOCIATIONS BETWEEN COGNITIVE AND MEMORY PROBLEMS, EMPLOYMENT AND QUALITY OF LIFE: A SURVEY OF EPILEPSY PATIENTS IN AUSTRALIA}

${ }^{1}$ Jeremy M Welton*, ${ }^{2}$ Christine Walker, ${ }^{3}$ Kate Riney, ${ }^{4}$ Alvin $\mathrm{Ng},{ }^{5}$ Lisa M Todd, ${ }^{6}$ Wendyl D'Souza. ' UCB Pharma, Melbourne, VIC, Australia; ${ }^{2}$ Chronic Illness Alliance, VIC, Australia; ${ }^{3}$ Queensland Children's Hospital, QLD, Australia; ${ }^{4}$ Costello Medical Singapore Pte Ltd, Singapore; ${ }^{5}$ Epilepsy Action Australia, Sydney, NSW, Australia; ${ }^{6}$ Department of Medicine, St Vincent's Hospital, The University of Melbourne, Melbourne, VIC, Australia

\subsection{6/jnnp-2019-anzan.85}

Introduction This analysis explored relationships between memory/cognitive issues, quality of life (QoL), and employment among patients with epilepsy (PwE) in Australia.

Methods Cross-sectional surveys were completed by PwE, or caregiver proxies, recruited via the online pharmacy application MedAdvisor and Australian PwE Facebook groups from May-August 2018. Data were collected on adverse events from antiepileptic drugs (AEDs), comorbidities, epilepsy severity and management, QoL (using QOLIE-10-P total score $)^{1}$ and demographics. Descriptive statistics were stratified by employment status: employed; not looking for work (NLW); looking for work (LW); or unable to work (UW), and differences in means or distributions were analysed using chisquared, Mann-Whitney $\mathrm{U}$ or Kruskal-Wallis $\mathrm{H}$ tests. Regression models were constructed to explore associations between memory/cognition-related variables and QOLIE-10-P within each employment group.

Results 950 eligible responses reporting current AED use were included $(71 \%$ via MedAdvisor, 29\% via Facebook; $55 \%$ seizure-free for $>1$ year). Mean QOLIE-10-P score was significantly different across employment groups $(\mathrm{p}<0.001)$ : 49.61 in employed PwE $(n=493), 48.87$ in NLW $(n=227)$, 32.75 in $\operatorname{LW~}(n=52)$, and 25.97 in UW $(n=178)$. After controlling for possible confounders, presence of memory problems from AEDs was associated with a -7.50 decrease in QOLIE-10-P only among employed PwE $(p=0.002)$. The extent that PwE felt bothered by memory difficulties, however, was significantly associated with QOLIE-10-P in all employment groups; generally, as level of concern about memory difficulties increased, estimated QOLIE-10-P decreased.

Conclusions Self-reported memory problems are prevalent among PwE in Australia and may impact QoL differentially depending on employment status. UCB Pharma-sponsored. 